\title{
Hypothermic Oxygenated Perfusion (HOPE) - A simple and effective method to modulate the immune response in kidney transplantation
}

\author{
Philipp Kron ${ }^{1,2 *}$, Andrea Schlegel ${ }^{1,3 *}$, Xavier Muller ${ }^{1}$, Ariana Gaspert ${ }^{4}$, \\ Pierre-Alain Clavien ${ }^{1}$, Philipp Dutkowski, MD $^{1}$
}

\footnotetext{
${ }^{1}$ Department of Surgery and Transplantation, Swiss HPB Centre, University Hospital Zurich, Switzerland

${ }^{2}$ HPB and Transplant Unit, St. James's University Hospital, Leeds, United Kingdom

${ }^{3}$ The Liver Unit, Queen Elizabeth Hospital Birmingham, Edgbaston, United Kingdom

${ }^{4}$ Department of Pathology and Molecular Pathology, University Hospital Zurich, Switzerland

Correspondence:

Philipp Dutkowski, MD

Department of Surgery and Transplantation

University Hospital Zurich

Raemistrasse 100, CH-8091 Zurich

Phone: +41 442554236

Fax: +41 442554999

E-mail: philipp.dutkowski@usz.ch
} 


\section{Figure S1. Experimental design.}

Four groups of kidneys were included in this experimental study (A). Allogeneic kidneys were either transplanted without any treatment of the recipients or following one-hour HOPE perfusion (B\&C). Such kidneys were compared to the standard application of Tacrolimus in recipients. Syngeneic kidneys from brown Norway rats were transplanted into brown Norway recipients and served as controls. Follow-up was 10 days. During this time several blood and plasma samples were obtained and assessed (D). Each group included 6 animals per time point, and 6-9 animals for the assessment of animal survival (E). Animals were randomly assigned to the experimental groups. 
Figure S1. Experimental design.

\section{A Experimental groups}

Group Donor Recipient

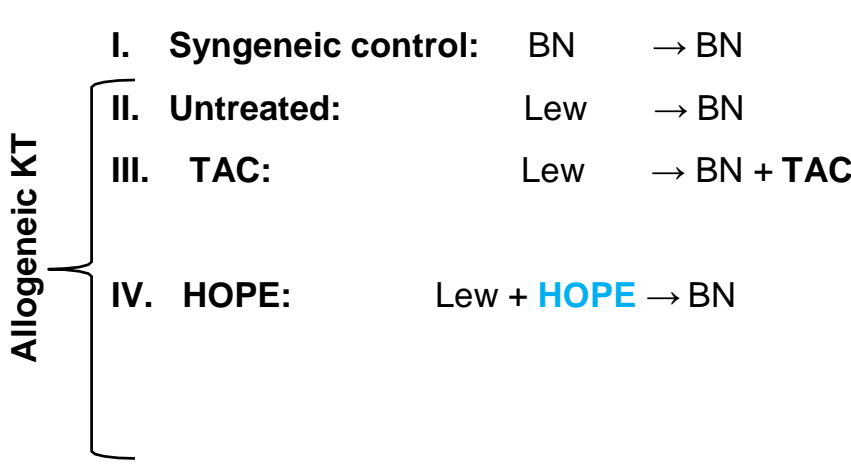

Rat strains:

TAC:

BN: Brown Norway; Lew: Lewis Tacrolimus

Laparotomy, ureter stent

kidney flush (saline $+1 \mathrm{U}$ Heparin/ $\mathrm{ml}, 20^{\circ}$ )

kidney flush and Stent placement for HOPE

Cuff placement during

minimal cold storage (10 $\mathrm{min})$

\section{Endpoints}

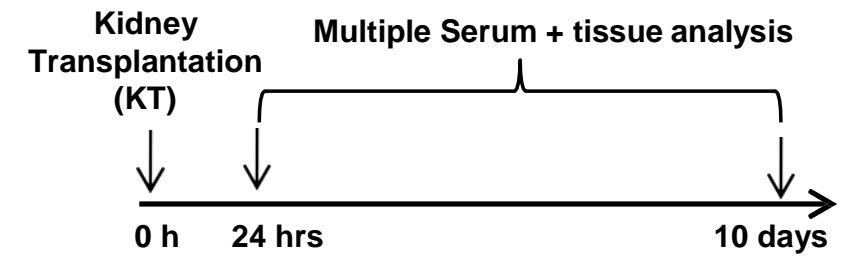

B Kidney grafts during preservation and at reperfusion (KT)
Donor kidney during Hypothermic oxygenated Cold storage
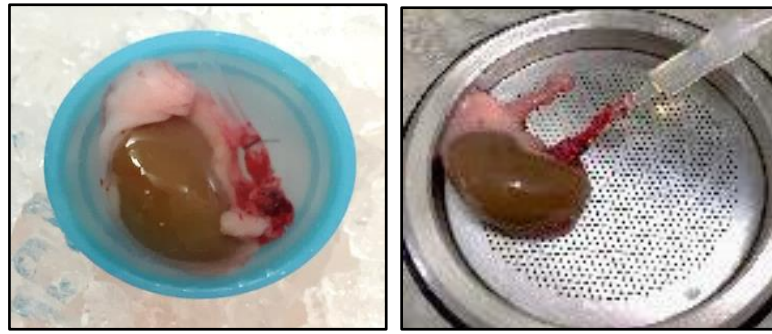

C Kidney grafts at procurement for analysis

Allogeneic untreated

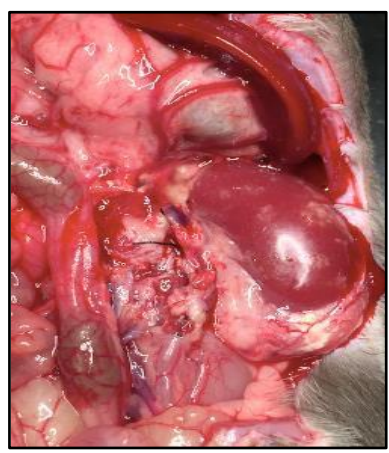

Allogeneic TAC

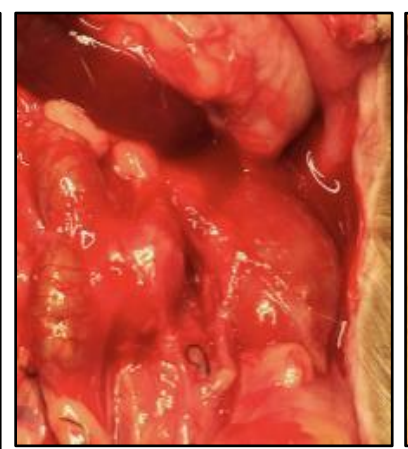

Kidney Reperfusion

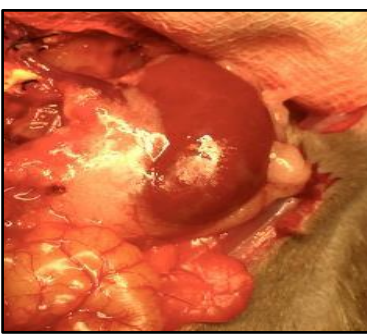

Allogeneic HOPE

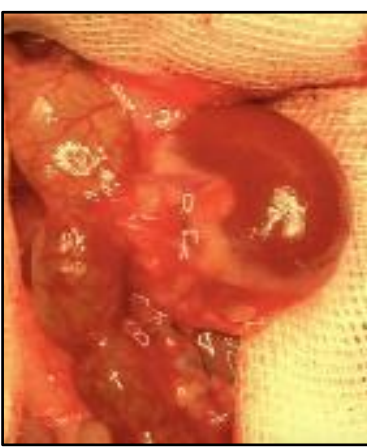

E Number of animals per group and time point (randomly assigned)

\begin{tabular}{|l|c|c|c|c|c|}
\hline Group (n)/Time points & 2 days & 4 days & $\mathbf{6}$ days & 8 days & 10 days \\
\hline . Syngeneic control & 6 & 6 & 6 & 6 & 7 \\
\hline II. Untreated & 6 & 6 & 6 & 6 & 7 \\
\hline III. TAC & 6 & 6 & 6 & 6 & 9 \\
\hline IV. HOPE & 6 & 6 & 6 & 6 & 7 \\
\hline
\end{tabular}

Experiments were terminated at each time point. 
A IL-6 Release
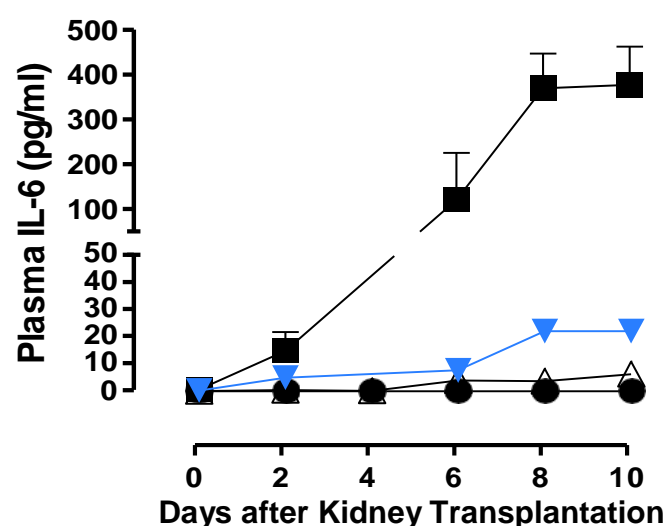

AUC at 10 days after KT: $p=0.0043$ (HOPE vs. untreated) $p=0.0022$ (untreated vs. TAC) $p=0.0043$ (TAC vs. HOPE)
B

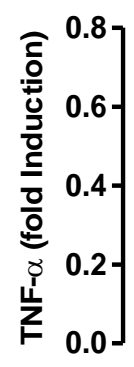$$
0
$$

Days after Kidney Transplantation

AUC at 10 days after KT: $p=0.0022$ (HOPE vs. untreated) $p=0.0022$ (untreated vs. TAC) $p=0.022$ (TAC vs. HOPE)
C

IL-10

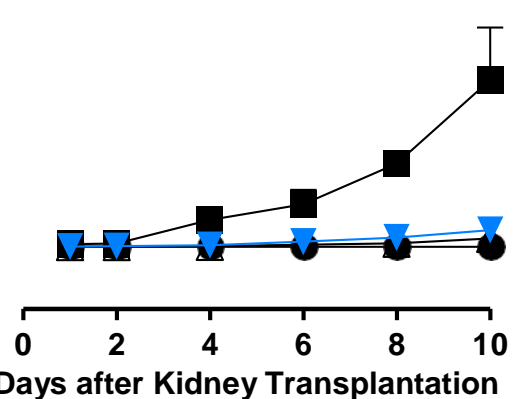

AUC at 10 days after KT: $p=0.0022$ (HOPE vs. untreated) $p=0.0012$ (untreated vs. TAC) $p=0.029$ (TAC vs. HOPE)

\section{Figure S2. Markers of reperfusion injury after KT.}

HOPE treatment was protective against up-regulation of several acute phase molecules in the setting of our allogeneic kidney transplant model. For example, the expression of IL-6, ENO, and TNF-alpha was significantly less compared to the cold storage group and remained comparable to the TAC group, for which recipients received additional Tacrolimus every day after kidney transplantation. 
Figure S3. Experimental design and ex vivo rat kidney perfusion and transplantation (DCD model).

\section{A Study groups}

1) No Injury (Baseline)

2) $D C D+C S$

3) $\mathrm{DCD}+\mathrm{CS}$ $+\mathrm{HOPE}$

4. $D C D+C S$ + HNPE

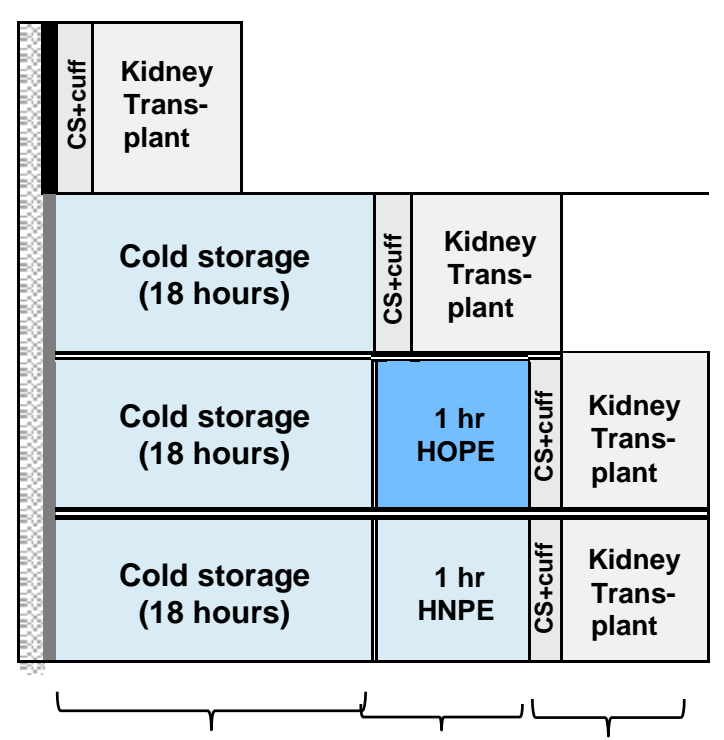

Initial Preservation Treatment Transplantation Injury

\section{B Endpoint}

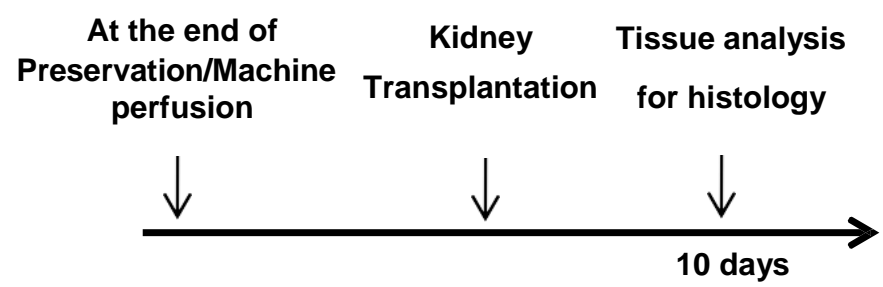

\section{Laparotomy, cannulation of ureter}

Kidney flush (saline + $1 \mathrm{U}$ Heparin $/ \mathrm{ml}, 20^{\circ}$ )

Kidney flush and Stent placement for Perfusion

\section{Figure S3. Experimental design (DCD model).}

Four groups of kidneys were included in this experimental study (A). Kidney donors underwent 30 minutes asystolic warm ischemia without previous heparinisation. Kidneys were flushed and retrieved and cold stored for 18 hours. During that time, kidneys were randomly assigned to the cold storage group or cold storage plus endischemic HOPE treatment or endischemic HNPE. All transplants in this set of experiments were from Brown Norway rat donors transplanted into Brown Norway recipients (syngeneic). The general follow-up was 10 days. Each group included 6 animals for that timepoint. Endpoints included staining for CD3, CD4, and CD8-positive cells on histological samples. 
Figure S4. T-cell activation after DCD KT.

A CD3 positive cells in kidney tissue

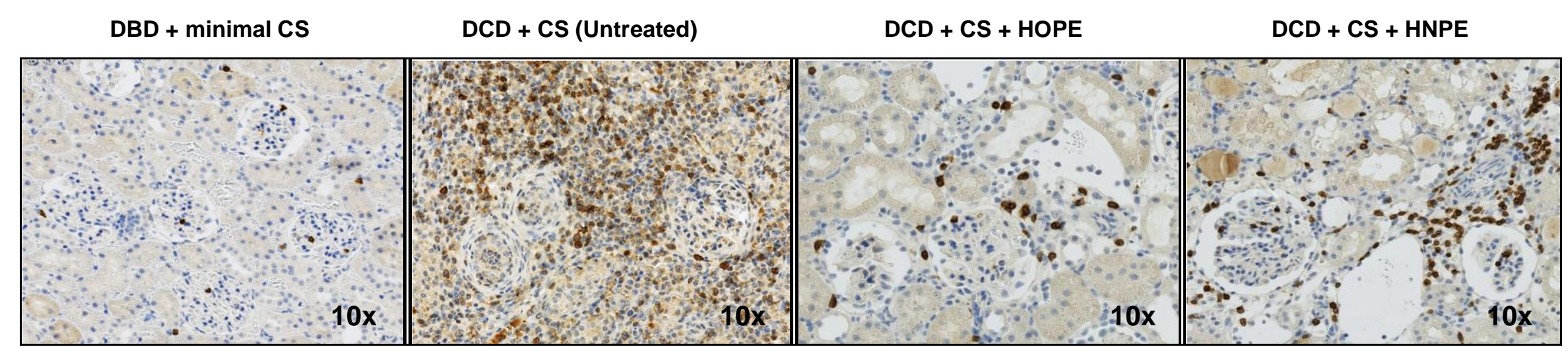

B CD4 positive cells in kidney tissue

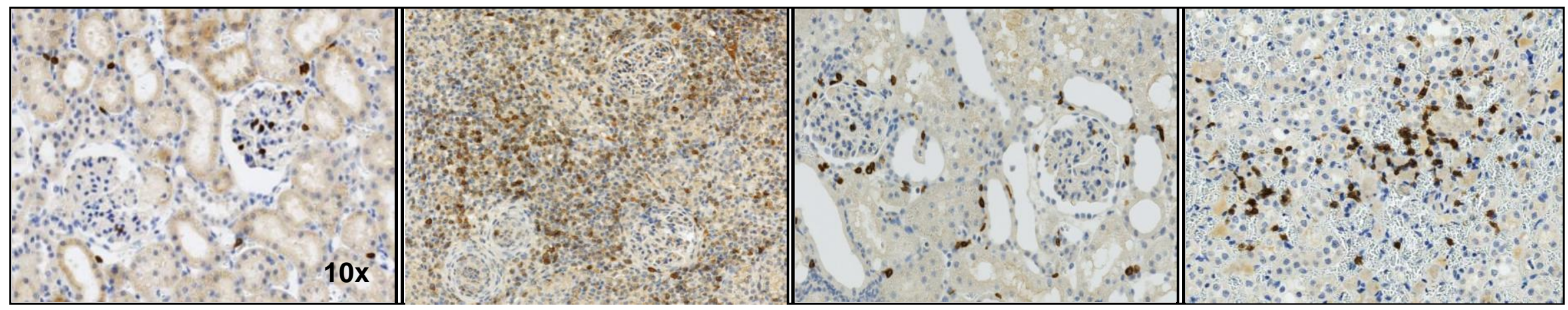

\section{CD8 positive cells in kidney tissue}

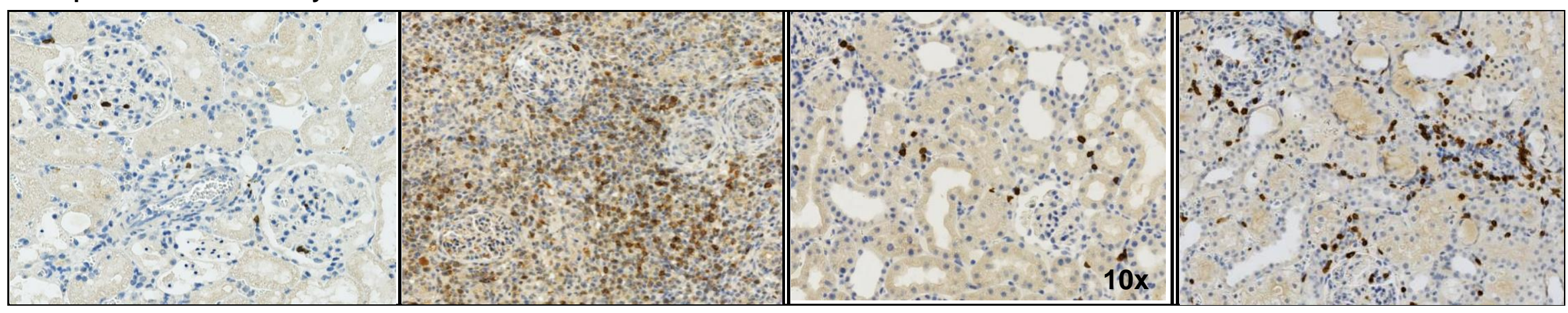


